\title{
Influencia del desarrollo urbano sobre el legado sostenible de los eventos deportivos internacionales
}

\author{
Influence of urban development on the sustainable legacy of international \\ sports events
}

\section{Darío José Espinal Ruiz , Claudia Fernanda Rojas Núñez², Miguel Fernando Reyes Velasco', Natali Cruz González ${ }^{1}$, Lina Marcela Vélez Sánchez ${ }^{2}$}

1 Facultad de Ciencias Económicas y de la Administración. Institución Universitaria Escuela Nacional del Deporte. Colombia.

2 Facultad de Ciencias de la Educación y el Deporte. Institución Universitaria Escuela Nacional del Deporte. Colombia.

CORRESPONDENCIA:

Darío José Espinal Ruiz

dario.espinal@endeporte.edu.co

Recepción: septiembre 2020 • Aceptación: mayo 2021
CÓMO CITAR EL ARTÍCULO:

Espinal-Ruiz, D., Rojas-Núñez, C., Reyes-Velasco, M., Cruz-Gonzáles, N., \& Vélez-Sánchez, L. (2021). Influencia del desarrollo urbano sobre el legado sostenible de los eventos deportivos internacionales. Cultura, Ciencia y Deporte, 16(50), 631-642. http:// dx.doi.org/ 10.12800/ccd.v16i50.1580

\section{Resumen}

Este estudio tiene como propósito identificar los efectos del legado de desarrollo urbano sobre el legado sostenible (económico, sociocultural, ambiental y deportivo) de los eventos deportivos internacionales. Se aplicaron 292 encuestas a los stakeholders directos de los eventos deportivos en Santiago de Cali- Colombia, utilizando un cuestionario estructurado en cinco constructos y 28 ítems. Se examinó la bondad de ajuste, la fiabilidad compuesta, la validez discriminante y el modelo estructural propuesto utilizando el método de modelado de ruta de mínimos cuadrados parciales (PLSSEM) en el software ADANCO. Los resultados indican que el legado de desarrollo urbano tiene una influencia significativa sobre los legados económico $(p=<.001)$, sociocultural $(p=<.001)$, ambiental $(p=<.001)$ y deportivo $(p=<.001)$. Este artículo contribuye a la literatura existente proporcionado conocimiento desde un enfoque cuantitativo sobre los factores que influyen en el legado sostenible de los eventos deportivos internacionales.

Palabras clave: desarrollo sostenible, desarrollo local, mínimos cuadrados parciales, legado de eventos deportivos.

\section{Abstract}

The purpose of this study is to identify the effects of the urban development legacy on the sustainable legacy (economic, sociocultural, environmental and sports) of international sports events. 292 surveys were applied to the direct stakeholders of the sporting events in Santiago de Cali- Colombia, using a questionnaire structured in five constructs and 28 items. Goodness of fit, composite reliability, discriminant validity and the proposed structural model were examined using the partial least squares (PLS-SEM) path modeling method in ADANCO software. The results indicate that the legacy of urban development has a significant influence on the economic $(p=<.001)$, sociocultural $(p=<.001)$, environmental $(p=<.001)$ and sports $(p=<.001)$ legacies. This article contributes to the existing literature providing knowledge from a quantitative approach on the factors that influence the sustainable legacy of international sports events.

Key words: sustainable development, local development, partial least squares, sports events legacy. 


\section{Introducción}

El análisis del legado de los eventos deportivos ha adquirido especial relevancia en los últimos años (Parra et al., 2016). Los eventos deportivos se han convertido en un elemento central en las prácticas gubernamentales que buscan generar beneficios positivos en el desarrollo local en el largo plazo (Thomson et al., 2019). El legado de un evento deportivo son los efectos planificados o no planificados que pueden ser positivos o negativos, tangibles o intangibles, que permanecen en el tiempo después de su conclusión (Preuss, 2007, 2015). La definición de legado va más allá del concepto tradicional de impacto, el cual se limita a la evaluación descriptiva de los resultados obtenidos por un evento deportivo tratado de forma aislada (Chalip, 2006). El legado de los eventos deportivos es una construcción social y política (Girginov, 2012a) que pretende brindar soluciones a los desafíos asociados a producir efectos positivos sostenibles (Byers et al., 2020), su gestión se realiza mediante la creación de políticas, programas y estructuras tangibles (Preuss, 2018) basadas en las necesidades y expectativas de sus diferentes stakeholders (Parent, 2016).

Los principales beneficios identificados en la literatura acerca del legado de los eventos deportivos se relacionan con la renovación urbana (Kassens-Noor et al., 2015), el impacto económico (Aguado et al., 2017), el desarrollo deportivo (Gratton \& Preuss, 2008), el turismo (Ferreira et al., 2018), el aumento de la participación deportiva (Chalip et al., 2017; Hyun \& Jordan, 2020) y los beneficios de carácter intangible, como el prestigio internacional, la felicidad y el orgullo de las comunidades anfitrionas (Hallmann et al., 2013; Maussier, 2017), entre otros. Sin embargo, la organización de un evento deportivo no garantiza efectos positivos per se (Müller, 2015). Las fallas en su organización pueden generar resultados contraproducentes en las finanzas públicas por sobrecostes (Flyvbjerg et al., 2020), la falta de apoyo popular (Preuss et al., 2020), la mala imagen internacional del país (Boykoff, 2017), la infraestructura deportiva infrautilizada (Mitrofanova et al., 2015), el incremento de las emisiones de gases efecto invernadero (Wicker, 2019), el desplazamiento urbano (Preuss, 2011), las tensiones ideológicas (Espinal-Ruiz et al., 2019) y la legitimidad gubernamental (Boykoff \& Zirin, 2016). Para mitigar los efectos negativos, los eventos deportivos deben ser sostenibles (Y.-D. Liu, 2018). El legado sostenible se refiere a mantener el legado positivo en el largo plazo de sus efectos directos e indirectos en los ámbitos social, económico y ambiental que justifiquen el uso de los recursos públicos, aumenten el apoyo popular y motiven a otras ciudades a albergar eventos futuros (Leopkey \& Parent, 2012).
Uno de los legados más importantes de los eventos deportivos es su capacidad para movilizar la transformación urbana (Latuf de Oliveira Sanchez \& Essex, 2018; Wilson, 2015). Los eventos deportivos son un poderoso mecanismo para acelerar este proceso, convirtiéndolos en el legado más esperado por las ciudades anfitrionas (Hiller, 2003a). La utilización de los eventos deportivos para el desarrollo urbano implica que su concepción debe ser económica, social y ambientalmente sostenible (Gaffney, 2013). Los comités organizadores, los gobiernos y las organizaciones deportivas han enfatizado cada vez más en la necesidad de que los eventos deportivos proporcionen un legado sostenible en las ciudades anfitrionas (Rojas \& Espinal-Ruiz, 2020). Sin embargo, la literatura actual sigue siendo limitada sobre la comprensión de este proceso (Kassens-Noor, 2016), especialmente en los países en desarrollo (Ferranti et al., 2020; Méndez, 2016; Toledo et al., 2015). Para contribuir a cerrar esta brecha, este estudio tiene como objetivo identificar los efectos del legado de desarrollo urbano sobre el legado sostenible (económico, sociocultural, ambiental y deportivo) de los eventos deportivos internacionales. La investigación fue realizada en Santiago de Cali, Colombia. Esta ciudad se caracteriza por organizar de manera permanente eventos deportivos de talla internacional que le han valido su reconocimiento como capital deportiva de América (Aces Europa, 2019).

\section{Revisión de la literatura y formulación de hipótesis}

Para comprender la percepción de los stakeholders directos de los eventos deportivos en Santiago de CaliColombia se utilizará la teoría del intercambio social, la cual ha sido empleada ampliamente para fundamentar estudios de este tipo (Karadakis \& Kaplanidou, 2012). De acuerdo a esta teoría, los stakeholders son más propensos a intercambiar con los demás si consideran que los beneficios obtenidos como resultado de la organización de eventos deportivos (Ej. Legado sostenible) son mayores a los efectos negativos que son difíciles de asumir (Ej. sobrecostes, desplazamiento urbano, entre otros) (Parra et al., 2016).

El legado sostenible es un concepto reciente en la literatura del sport management, proviene del propósito del Comité Olímpico Internacional para que los Juegos Olímpicos proporcionen efectos económicos, sociales y ambientales positivos en el largo plazo en las ciudades anfitrionas (Kim et al., 2019). Girginov y Hills (2008) sugieren que el legado sostenible es un concepto ambiguo, ya que intenta satisfacer la necesidad de crecimiento acelerado, al tiempo que promueve valores de igualdad, solidaridad y responsabilidad. Asegu- 
rar el legado sostenible de un evento deportivo exige desarrollar un proceso de planeación de largo plazo basado en un modelo de gobernanza fundamentado sobre la visión compartida de los stakeholders acerca de las transformaciones que se pretenden lograr en las ciudades anfitrionas (Holmes et al., 2015). Esto implica que, para maximizar el legado sostenible positivo de un evento deportivo, el apoyo de sus stakeholders es esencial (Karadakis \& Kaplanidou, 2012). Por tanto, es necesaria su articulación en una red de gobernanza responsable de dirigir la acción colectiva hacia el logro del legado sostenible (Girginov, 2012b) que incluya: al comité organizador local, al cuerpo de voluntarios, al gobierno local, la comunidad, las organizaciones deportivas, las delegaciones, los medios de comunicación y los patrocinadores, entre otros (Parent \& SmithSwan, 2012).

El legado de los eventos deportivos es un concepto multidimensional (Chappelet, 2012). Los elementos clave a considerar en este enfoque incluyen el legado sociocultural, socioeconómico y el ambiental (Girginov \& Hills, 2009). Leopkey y Parent (2012) identificaron los legados de tipo cultural, económico, ambiental, imagen, informativo/educativo, nostalgia, movimiento olímpico, físico, político, psicológico, social, deportivo, sostenible y el legado urbano. Grix et al., (2017) sugieren que los legados se clasifican en económico, regeneración urbana, orgullo/bienestar, mayor participación en la actividad física, prestigio internacional y soft power. Chappelet (2012) propone la matriz de legados deportivos/no deportivos; territoriales/personales y tangibles/intangibles. Cashman (2003) identifica el legado económico, infraestructura física, educación, político, cultural, deportivo y memoria histórica. Hiller (2003b) propone los legados de desarrollo urbano, desarrollo económico, memoria histórica y comunidades. Gratton y Preuss (2008) sugieren los legados de infraestructura, conocimiento, desarrollo de capacidades y educación, imagen, emociones, redes y cultural. Mientras que, el Comité Olímpico Internacional (2017) resalta los legados deportivos, sociocultural, ambiental, económico y desarrollo urbano. Basándose en la evidencia citada, la operacionalización propuesta en este artículo pretende investigar cómo el legado de desarrollo urbano influye sobre el legado sostenible (sociocultural, economico, ambiental y deportivo) de los eventos deportivos.

\section{Desarrollo urbano y legado sostenible}

Los eventos deportivos son considerados una poderosa herramienta para emprender planes de desarrollo urbano, aspectos como la infraestructura deportiva, la renovacion urbana, la mejora de los sistemas de transporte y del espacio público contribuyen a construir ciudades más sostenibles, seguras y resilientes (Ferranti et al., 2020; Sánchez, 2019). Las transformaciones urbanas requieren grandes inversiones para producir el legado esperado por las ciudades anfitrionas (Cashman, 2011). En este sentido, el legado económico resalta la oportunidad de atraer nuevas inversiones públicas y privadas para promover el desarrollo local con proyectos de transformación del espacio urbano (Leal de Oliveira et al., 2020). Estos desafíos se han visto acentuados por el papel que el diseño y la arquitectura de infraestructura deportiva ha asumido en la transformación urbana (Latuf de Oliveira Sanchez \& Essex, 2018). Por lo tanto, se propone la siguiente hipótesis:

H1. El legado de desarrollo urbano tiene un impacto directo y positivo sobre el legado económico de los eventos deportivos.

El desarrollo urbano deportivo es uno de los principales movilizadores sociales del deporte (Coalter, 2007). El desarrollo urbano deportivo debe planificarse y gestionarse si se buscan legados sociales sostenibles (Añó et al., 2012; Smith, 2009, 2010). La construcción de infraestructura con un sentido de conciencia comunitaria aumenta los beneficios relacionados a la utilización del espacio público, aumenta el capital social y promueve una ciudadanía activa (Pye et al., 2015). Se puede evidenciar que el legado del desarrollo urbano e infraestructura deportiva tiene una influencia significativa sobre el desarrollo sociocultural de las comunidades (Wilson, 2015). Por lo tanto, se propone la siguiente hipótesis:

H2. El legado de desarrollo urbano tiene un impacto directo y positivo sobre el legado sociocultural de los eventos deportivos.

Existe una relación recíproca entre el deporte y el medio ambiente (Orr \& Inoue, 2019). La investigación ha demostrado algunos efectos negativos de los eventos deportivos sobre el medio ambiente (Wicker, 2019). Como resultado, la preocupación por el legado ambiental ha sido parte importante en la organización de eventos deportivos (Chappelet, 2008). Actividades concernientes a la construcción, renovación y uso de la infraestructura requerida para los eventos deportivos generan efectos ambientales (Collins et al., 2007). Estudios recientes revelan cómo se ha venido consolidando en el tiempo la relación entre el desarrollo urbano y la construcción de legado ambiental (Azzali, 2019). Por lo tanto, se propone la siguiente hipótesis: 
H3. El legado de desarrollo urbano tiene un impacto directo y significativo sobre el legado ambiental de los eventos deportivos.

La investigación ha demostrado que la organización de eventos deportivos tiene efectos temporales sobre el legado deportivo (Pappous, 2011; Taks et al., 2017). Sin embargo, se han identificado relaciones positivas entre las facilidades de acceso público a las instalaciones deportivas y el incremento de la participación deportiva de las comunidades aledañas (Chalip et al., 2017; Kristiansen, 2017), siendo un factor determinante en el cambio positivo del comportamiento frente los hábitos de vida saludable (Pye et al., 2015). La evidencia sugiere que la inversión en desarrollo urbano y la infraestructura deportiva de los eventos deportivos genera efectos positivos sobre el legado deportivo (Gehl, 2010; Wilson, 2015). Por lo tanto, se propone la hipótesis:

H4. El legado de desarrollo urbano tiene un impacto directo y significativo sobre el legado deportivo de los eventos deportivos.

\section{Método}

\section{Contexto del estudio}

La ciudad de Santiago de Cali es la capital del Departamento del Valle del Cauca, ubicado al suroccidente colombiano. Es la tercera economía del país de vocación principalmente agroindustrial y de servicios. La ciudad ha albergado numerosos eventos deportivos de nivel internacional como los Juegos Panamericanos de 1971; en el 2011 fue sede de la Copa Mundo de la FIFA sub-20; en el 2013 de los Juegos Mundiales; en el año 2014 organizó el Campeonato Mundial de Ciclismo (del cual ha sido sede en 13 oportunidades); en el 2015 fue sede del Campeonato Suramericano de Menores de Atletismo, del IX Campeonato Mundial de Atletismo de Menores de la IAAF, el Campeonato Sudamericano de Voleibol Femenino, y del LX Campeonato Mundial FIRS-Cali 2015 de patinaje artístico. Para el año 2016, se organizaron trece eventos internacionales, entre los más relevantes se encuentran: el Mundial de Clubes de Fútbol de Salón, la Copa Mundo de Futsal de la FIFA y el Campeonato Mundial de Raquetball. Además, Santiago de Cali fue seleccionada como sede de los primeros juegos Panamericanos Juveniles para el año 2021 y sede del campeonato mundial de atletismo sub-20 en el 2022 y sede de Copa América de Fútbol. Como resultado de la experiencia en organización de eventos deportivos, su infraestructura deportiva, la oferta pública de programas deportivos y su reconocida tradición internacional obtuvo el reconocimiento como la capital deportiva de América en el año 2019 (Aces Europa, 2019).

\section{Participantes}

La revisión de la literatura muestra que uno de los principales propósitos de los eventos deportivos es la creación de una variedad de beneficios para sus stakeholders (Leopkey \& Parent, 2015). Dado que este estudio examina la influencia del legado sostenible de los eventos deportivos, los sujetos seleccionados en esta investigación son los stakeholders que pertenecen a organizaciones de tipo: cultural (2\%) comunitario (7\%), deportivo (63\%), educativo (12\%), sector empresarial (11\%) y sector público (5\%) de Santiago de Cali, Colombia (Tabla 1). Se adoptó la técnica de la encuesta basada en cuestionarios estructurados utilizando un muestreo por conveniencia. Este tipo de muestreo fue seleccionado debido a que el PLS-SEM al ser un método basado en la varianza no requiere de muestreo probabilístico (Henseler et al., 2015). Se aplicaron un total de 292 encuestas de manera online, lo cual cumple con el estándar sugerido para la metodología PLS-SEM.

Tabla 1. Características de la muestra.

\begin{tabular}{lcc}
\hline Características & Frecuencias & Porcentaje \\
\hline Género & 168 & 57.5 \\
Mujer & 122 & 42.5 \\
Hombre & & \\
\hline Edad (años) & 47 & 16 \\
$19-25$ & 65 & 22 \\
$26-35$ & 43 & 15 \\
$36-45$ & 73 & 25 \\
$46-55$ & 64 & 22 \\
Más de 55 & & \\
\hline Nivel de educación & 40 & 13 \\
Secundaria & 147 & 51 \\
Universitario & 105 & 36 \\
Posgradual & & \\
\hline Tiempo de residencia (años) & 10 & 4 \\
Menos de 1 & 8 & 3 \\
1-3 & 14 & 5 \\
$4-10$ & 26 & 99 \\
11-20 & 233 & \\
Más de 20 & & \\
\hline
\end{tabular}

Fuente: elaboración propia.

\section{Instrumento}

Se utilizó un cuestionario estructurado en 28 ítems sobre legado de eventos deportivos. Para el legado sociocultural, legado económico, legado ambiental, legado desarrollo urbano y legado deportivo; los constructos y sus ítems fueron adaptados del trabajo previo 
de Parra et al., (2016), Ma et al., (2011) y Liu et al., (2014). Todos los constructos y sus respectivos ítems fueron operacionalizados en una escala Likert de 5 puntos en la que: 1 , totalmente en desacuerdo y 5 , totalmente de acuerdo (ver Apéndice 1). El cuestionario también incluía otras variables de control que describen las características sociodemográficas de la muestra como edad, género, nivel de educación y tiempo de residencia en la ciudad.

\section{Procedimiento y análisis de datos}

Este estudio se realizó siguiendo el método de ecuaciones estructurales de mínimos cuadrados parciales (PLS-SEM), donde se propuso un modelo de segundo orden de tipo reflectivo. Se seleccionó este método debido a que permite modelar y construir conceptos (Höök \& Löwgren, 2012), lo que lo convierte en una técnica ideal para el estudio de factores de éxito permitiendo contrastar un modelo hipotético contra los datos (Albers, 2010). El método PLS-SEM fue elegido con base en las premisas de Aldás (2016), quien sugiere que este enfoque es útil cuando el objeto de investigación considera un modelo hipotético que aún no ha sido validado empíricamente (Chin et al., 2003), permitiendo examinar el poder predictivo que tienen las variables latentes exógenas sobre la variable latente endógena (Hair et al., 2011). Una de las ventajas de este enfoque es que la distribución de la población no está sujeta a restricciones, por lo que se pueden realizar investigaciones exploratorias y confirmatorias con muestras pequeñas y medianas (Tsao et al., 2016).

Para el análisis de las variables sociodemográficas se utilizó el software SPSS versión 20.0 en el cálculo de las frecuencias y porcentajes. Para el análisis de datos, se utilizó el software ADANCO versión 2.2.0 (Henseler \& Dijkstra, 2020), siguiendo la metodología de Henseler et al., (2016). El primer paso para validar el modelo de medida fue analizar la validez de contenido por medio de la revisión y adaptación de escalas de modelos de evaluación ya validados en la literatura. Esto se corroboró con la realización de un estudio piloto con 30 muestras que permitió asegurar las propiedades estadísticas de las medidas y evaluar la validez aparente y de contenido de los constructos. Seguido, se analizó la bondad de ajuste del modelo (SRMR <.08), la confiabilidad compuesta (AVE >.5; Dijkstra-Henseler's rho >.7; Cronbach's >.7), la validez discriminante (FornellLarcker, HTMT <1), y el análisis del modelo estructural para la prueba de hipótesis a través de la prueba bootstrap del ajuste general del modelo $\left(\mathrm{R}^{2}\right.$; Path $\beta$; SE; $t$-value; $p$ value; Cohen's f2). Todos los valores representados demostraron la validez del modelo sugerido.

\section{Resultados}

\section{Confiabilidad y validez del modelo}

Antes de examinar la medida y el modelo estructural, se debe realizar la evaluación de la bondad del ajuste del modelo (Henseler et al., 2016). Para ello, el software ADANCO proporciona la Raíz Cuadrática Media Estandarizada (SRMR) como la medida de ajuste del modelo. De acuerdo con Hair et al., (2014) y Hu y Bentler (1998), un valor inferior a 0 o .08 es una buena opción para SRMR. Para este estudio, el resultado del SRMR saturado fue de .0630, lo que indica que el modelo cumple con este criterio.

Para garantizar la confiabilidad del modelo, este estudio considera la confiabilidad compuesta. Se analizó la varianza promedio extraída (validez convergente AVE), $\alpha$ de Cronbach (validez interna del modelo) y la prueba de $\rho$ A Dijkstra-Henseler (Fiabilidad de las puntuaciones de constructo) (Henseler et al., 2016). Todos los valores de las cargas factoriales, $\rho$ A y $\alpha$ de Cronbach son aceptables (>.7), justificando la fiabilidad de los constructos. Además, los valores de AVE para todos los constructos denotan la validez convergente, ya que los valores están por encima del nivel mínimo requerido de .50 (Tabla 2).

Para evaluar la validez discriminante entre los constructos se utilizaron los criterios de Fornell y Larcker (1981) de carga cruzada y la Heterotrait-Monotrait (HTMT). En la prueba Fornell- Larcker las cargas de un indicador en su propia variable son, en todos los casos, mayor que todas sus cargas cruzadas con otras variables, por ende, la prueba es válida (Tabla 3).

Tabla 2. Confiabilidad compuesta del modelo.

\begin{tabular}{lcccc}
\hline Constructos & Items & AVE $>.5$ & Dijkstra-Henseler's $(\boldsymbol{\rho})(>.7)$ & .9156 \\
\hline Legado socioeconómico & 7 & .6645 & .9022 & .9155 \\
Legado sociocultural & 7 & .6248 & .8602 & .8992 \\
Legado desarrollo urbano & 6 & .5848 & .8550 & .8575 \\
Legado deportivo & 5 & .6287 & .9032 & .8514 \\
Legado ambiental & 3 & .8332 & .9000 \\
\hline
\end{tabular}

Fuente: autores-ADANCO. 
Tabla 3. Validez discriminante- criterio de Fornell-Larcker.

\begin{tabular}{|c|c|c|c|c|c|}
\hline Constructos & SE & SC & DU & LD & LA \\
\hline Legado socioeconómico (SE) & .6645 & & & & \\
\hline Legado sociocultural (SC) & .3004 & .6248 & & & \\
\hline Legado desarrollo urbano (DU) & .4744 & .4646 & .5848 & & \\
\hline Legado deportivo (LD) & .2822 & .5635 & .5428 & .6287 & \\
\hline Legado ambiental (LA) & .1909 & .3362 & .3599 & .3085 & .8332 \\
\hline
\end{tabular}

Fuente: autores-ADANCO.

Tabla 4. Validez discriminante-HTMT.

\begin{tabular}{lllll}
\hline Constructos & SE & SC & DU & LD \\
\hline Legado socioeconómico (SE) & & & & \\
Legado sociocultural (SC) & .6030 & & \\
Legado desarrollo urbano (DU) & .7755 & .7707 & .8569 \\
Legado deportivo (LD) & .5950 & .8570 & .659 \\
Legado ambiental (LA) & .4778 & .6393 & .6761 & .6337 \\
\hline
\end{tabular}

Fuente: autores-ADANCO

Tabla 5. Modelo estructural y pruebas de hipótesis.

\begin{tabular}{lcccccc}
\hline Hipótesis & Path $\boldsymbol{\beta}$ & SE & t-value & Cohen's $\mathbf{f}^{2}$ & $\boldsymbol{p}$-value & Apoyada \\
\hline H1. Legado desarrollo urbano $->$ Legado económico & .689 & .043 & 16.012 & .902 & $* * *$ & Apoyada \\
H2.Legado desarrollo urbano $->$ Legado sociocultural & .682 & .037 & 18.220 & .867 & $* * *$ & Apoyada \\
H3. Legado desarrollo urbano $->$ Legado ambiental & .600 & .038 & 15.481 & .562 & $* * *$ & Apoyada \\
H4. Legado Desarrollo urbano -> Legado deportivo & .737 & .027 & 27.064 & 1.187 & $* * *$ & Apoyada \\
\hline
\end{tabular}

Nota: *t-values: 1.65 (10\%); **t-values: 1.96 (5\%); ***t-values: $2.58(1 \%)$

Nota: Path $\beta * p=<.05 ;{ }^{*}{ }^{*} p=<.01 ; * * * p=<.001$.

Nota: Cohen's f2: .35 (fuerte), .15 (moderado); .02 (aceptable).

De igual manera, Henseler et al., (2015) indican que se requiere una relación de las correlaciones Heterotrait-Monotrait (HTMT) para examinar la validez discriminante en el enfoque PLS-SEM, la cual debe ser menor de.9 (Teo et al., 2008). Todos los valores HTMT están por debajo del valor requerido. Por lo tanto, se cumple la validez discriminante de todos los constructos (Tabla 4).

\section{Modelo estructura}

Para probar las hipótesis del modelo estructural, los resultados proporcionados por la técnica de bootstrapping en ADANCO se realizaron a través de 4.999 remuestreos (Henseler et al., 2016). Las estadísticas proporcionadas por los resultados del análisis incluyen el coeficiente de ruta (path $\beta$, el error estándar (SE), el valor $t$, Sig., $\mathrm{f}^{2}$ de Cohen, $\mathrm{R}^{2} \mathrm{y} \mathrm{R}^{2}$ ajustado). Para definir el poder predictivo del modelo, Chin (1998) considera valores en el $\mathrm{R}^{2}$ de: .67 (sustancial); .33 (moderado), y; .10 (débil), respectivamente. De acuerdo con esto, la varianza explicada indica que el legado de desarrollo urbano predice moderadamente los constructos de legado económico $\left(\mathrm{R}^{2}=.4744\right.$; $\mathrm{R}^{2}$ ajustado $\left.=.4726\right)$, legado sociocultural $\left(\mathrm{R}^{2}=.4646\right.$;

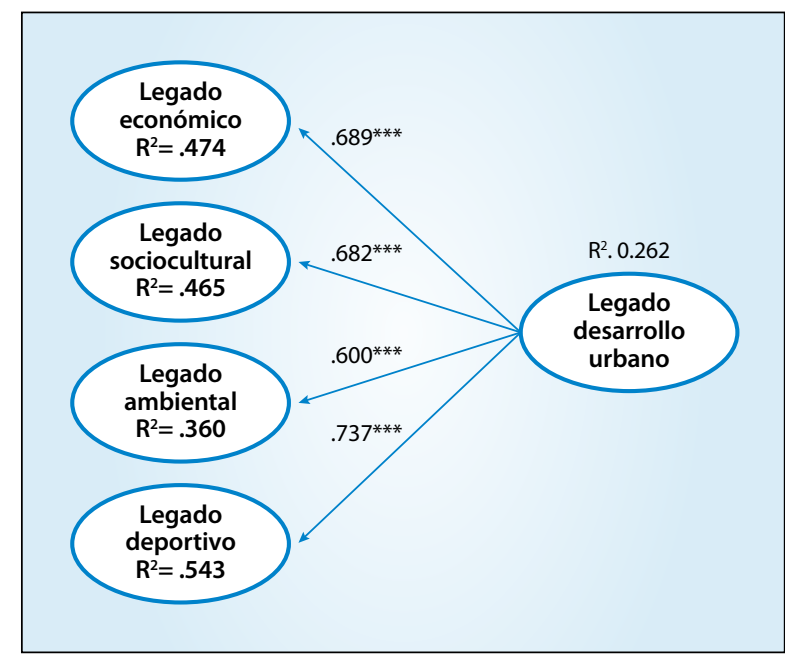

Figura 1. Modelo estructural.

$\mathrm{R}^{2}$ ajustado $\left.=.4628\right)$, legado ambiental $\left(\mathrm{R}^{2}=.3599 ; \mathrm{R}^{2}\right.$ ajustado $=.3577)$ y legado deportivo $\left(\mathrm{R}^{2}=.5428 ; \mathrm{R}^{2}\right.$ ajustado $=.5412)$ (Figura 1$)$. En la tabla 5 se muestran los resultados detallados del bootstraping para las pruebas de hipótesis.

Las rutas que apoyan las hipótesis del estudio ( $\mathrm{f}^{2} \mathrm{de}$ cohen) son superiores que .35 , lo que demuestra que el tamaño de sus efectos son fuertes (Cohen, 1988). Esto 
sumado a que todos los valores $t$ son superiores a 2.58, corroborando la precisión de las estimaciones (Valaei \& Jiroudi, 2016), por lo tanto, todas las hipótesis propuestas en el estudio son apoyadas.

\section{Discusión}

Este artículo explora el fenómeno del legado de los eventos deportivos aplicando un modelo de ecuaciones estructurales siguiendo la metodología PLS-SEM para poner a prueba los efectos del desarrollo urbano sobre las dimensiones del legado sostenible. Mientras que existe un cuerpo creciente de literatura sobre el legado de eventos deportivos, el análisis de las relaciones causales entre sus dimensiones constitutivas ha sido poco explorado. Para ayudar a cerrar esta brecha, este estudio contribuye de manera preliminar presentando un modelo empírico que permite analizar la percepción de los stakeholders directos de los eventos deportivos. En términos generales, los resultados de investigación indican que, según la percepción de los stakeholders, existen relaciones significativas entre el desarrollo urbano y el legado sostenible de los eventos deportivos internacionales. Esto se considera un avance relevante ya que brinda evidencias empíricas sobre cómo los eventos deportivos permiten construir ciudades más sostenibles (Ferranti et al., 2020; Sánchez, 2019).

El legado de desarrollo urbano asociado a la organización de eventos deportivos internacionales tiene una influencia significativamente positiva sobre el legado económico. Este resultado es coherente con lo propuesto por Cashman (2011); se puede evidenciar que las grandes inversiones públicas y privadas en infraestructura aportan beneficios económicos a las ciudades anfitrionas. Los eventos deportivos se convierten en un mecanismo para promover el desarrollo económico local por medio de la transformación urbana (Leal de Oliveira et al., 2020), la mejora de los sistemas de transporte, el incremento del comercio, el turismo, el empleo y el consumo interno son algunos de sus efectos más importantes.

El legado de desarrollo urbano asociado a la organización de eventos deportivos internacionales tiene una influencia significativamente positiva sobre el legado sociocultural. Esto implica que las inversiones en desarrollo urbano e infraestructura deportiva son buenos movilizadores de la ciudadanía activa (Pye et al., 2015) y el desarrollo sociocultural de las comunidades (Wilson, 2015), promoviendo el orgullo ciudadano, la cohesión, la solidaridad y la interculturalidad (Parra et al., 2016). Este resultado se alinea con lo propuesto por Coalter (2007), proporcionando evidencia empírica sobre el desarrollo urbano deportivo como detonante de los beneficios sociales del deporte.

Se verifica que el legado de desarrollo urbano asociado a la organización de eventos deportivos internacionales tiene una influencia significativamente positiva sobre el legado ambiental. Este resultado afirma lo expuesto por Collins et al. (2008), evidenciando que la construcción y renovación urbana y de infraestructura deportiva genera efectos ambientales. En consecuencia, la preocupación por el medio ambiente resulta crucial para que los eventos deportivos generen efectos positivos sobre las ciudades anfitrionas (Azzali, 2019), especialmente en lo relacionado a la protección del agua, el aire, la biodiversidad y el patrimonio arquitectónico.

Los resultados indican que el legado de desarrollo urbano asociado a la organización de eventos deportivos internacionales tiene una influencia significativamente positiva sobre el legado deportivo. La facilidad de acceso de la ciudadanía a espacios de práctica contribuye a incrementar los niveles de participación deportiva de las comunidades aledañas (Chalip et al., 2017; Kristiansen, 2017). Esto sumado a procesos de transformación urbana relacionada a la renovación del espacio público generan efectos positivos sobre los hábitos de práctica deportiva en las ciudades anfitrionas (Gehl, 2010). El acceso a la infraestructura deportiva, el crecimiento de las organizaciones deportivas, el aumento de las oportunidades a los jóvenes y la participación ciudadana son algunos de los efectos con mayor relevancia.

\section{Conclusiones}

El constructo legado en el desarrollo urbano tiene una influencia directa y positiva sobre los constructos legado sociocultural, legado socioeconómico, legado ambiental y legado deportivo. Por lo tanto, las hipótesis H1, H2, H3 y H4 son apoyadas. Este estudio desarrolló y probó empíricamente un modelo teórico que tiene como objetivo analizar la influencia del legado de desarrollo urbano e infraestructura sobre el legado sostenible de los eventos deportivos internacionales. El modelo propuesto comprobó que existen relaciones directas y positivas entre el legado de desarrollo urbano y los legados económicos, socioculturales, ambientales y deportivos. En consecuencia, se puede afirmar que el legado de desarrollo urbano es un buen predictor del legado sostenible en ciudades anfitrionas de eventos deportivos internacionales. Este resultado confirma las conclusiones de la literatura previa en 
sport management en lo relacionado a que los eventos deportivos son un mecanismo legítimo para promover el desarrollo local sostenible.

Aunque el concepto de legado ha sido ampliamente explorado en la literatura, aún no existe consenso acerca de su definición y modelos de medición. Esto se evidencia en la diversidad de posturas que los autores sugieren como sus dimensiones constitutivas. No obstante, este estudio es una valiosa contribución al campo del análisis de legado de los eventos deportivos introduciendo un modelo predictivo que permite identificar relaciones causales entre los diferentes factores que se han establecido como relevantes para promover el legado sostenible. Sin embargo, los autores reconocen que no sería posible construir un modelo que fuera totalmente válido cuando se intenta estudiar patrones de causalidad en un sistema complejo que cuenta con un gran número de variables que no se pueden controlar. A pesar de las limitaciones de este artículo, la evidencia empírica presentada ofrece respuestas preliminares que permiten analizar el fenómeno del legado sostenible en el contexto de una ciudad en desarrollo con amplia experiencia en organizar eventos deportivos de talla internacional.

Este estudio tiene importantes implicaciones prácticas para investigadores, gestores deportivos y formuladores de políticas de desarrollo local. Para garantizar el legado sostenible de los eventos deportivos se debe prestar atención en la planificación de los diferentes legados en una etapa post evento. Esto implica que se deben articular redes de gobernanza que representen los diferentes stakeholders directos que velen por asegurar los efectos positivos en la economía, la sociedad, el medio ambiente y el deporte. La connotación de organizar un evento deportivo trasciende hacia la ga- rantía de asegurar que su legado contribuya a los $\mathrm{Ob}$ jetivos del Desarrollo Sostenible 2030, proceso que se convierte en un círculo virtuoso cuando se disminuye el riesgo de fracaso en las grandes inversiones públicas y privadas necesarias para su ejecución, aumenta el apoyo popular y motiva a otras ciudades a albergar eventos deportivos.

Aunque este estudio proporciona hallazgos significativos sobre los factores que influyen en el legado sostenible de eventos deportivos, persisten algunas limitaciones, lo que suministra las direcciones para la investigación futura. En primer lugar, los resultados de investigación se limitan a la percepción de los stakeholders directos de los eventos deportivos en el contexto de Santiago de Cali (Colombia). Además, el tipo de muestreo utilizado (conveniencia) no permite la generalización de los resultados a toda la población objeto de estudio. La investigación futura debería replicar este estudio en otras regiones y en otros países que permitan las comparaciones en contextos culturales y económicos diversos utilizando muestreos probabilísticos. En segundo lugar, este estudio es cross-sectional, por lo tanto no se considera cómo ha evolucionado el sistema. La investigación futura debería considerar realizar un estudio longitudinal que permitiera comparar la percepción de los stakeholders en diferentes periodos de tiempo. A pesar de que se consideran múltiples constructos referenciados en la literatura que analizan el legado de los eventos deportivos, es posible que algunos factores hayan sido omitidos. Por lo tanto, la investigación futura debería considerar integrar factores en el modelo para mejorar su poder predictivo, tales como prestigio internacional, soft power, la felicidad y el orgullo de las comunidades anfitrionas, entre otros. 


\section{Apéndice 1. Constructos e ítems.}

\section{Legado desarrollo urbano}

1. Los eventos deportivos internacionales han mejorado la infraestructura de la ciudad (accesos, calles, mobiliario urbano...).

2. Los eventos deportivos internacionales han permitido mejorar el sistema de transporte público en la ciudad.

3. Las infraestructuras creadas para los eventos deportivos internacionales han sido provechosas para la ciudadanía.

4. Los eventos deportivos internacionales han permitido regenerar las infraestructuras de la zonas aledañas donde se han celebrado (calles, iluminación, embellecimiento....).

5. Los eventos deportivos internacionales han permitido mejorar los servicios sociales en la ciudad (centros de salud, educación, etc.).

6. Las infraestructuras creadas para los eventos deportivos internacionales son útiles para otras actividades.

\section{Legado económico}

7. Los eventos deportivos internacionales han aportado beneficios económicos a la ciudad.

8. Los eventos deportivos internacionales han aumentado el turismo en la ciudad durante todo el año.

9. Los eventos deportivos internacionales han incrementado el comercio y los negocios en la ciudad.

10. Los eventos deportivos internacionales han incrementado las inversiones privadas y las oportunidades de negocio en la ciudad.

11. Los eventos deportivos internacionales han incrementado las oportunidades para trabajar en la ciudad.

12. Los eventos deportivos internacionales han incrementado el consumo de bienes y servicios en la ciudad.

13. Los eventos deportivos internacionales han aportado grandes beneficios económicos a los barrios de la zona donde se han celebrado.

\section{Legado socio cultural}

14. La celebración de eventos deportivos internacionales ha incrementado el orgullo de los ciudadanos por vivir en la ciudad.

15. La celebración de eventos deportivos internacionales han permitido un mayor conocimiento del movimiento Olímpico.

16. Los eventos deportivos internacionales han incrementado la cohesión y la unidad de la ciudadanía.

17. Los eventos deportivos internacionales han proporcionado a los ciudadanos una oportunidad para conocer a nuevas personas.

18. Los eventos deportivos internacionales han mejorado la solidaridad y la hospitalidad de los ciudadanos con los turistas

19. Los eventos deportivos internacionales han favorecido el intercambio cultural y la comprensión de otras culturas.

20. Los eventos deportivos internacionales han contribuido a la preservación, la conservación y la promoción de la cultura ciudadana.

Legado ambiental

21. Los eventos deportivos internacionales se han preocupado por proteger el medio ambiente de la ciudad (residuos, agua, aire, etc.).

22. Los eventos deportivos internacionales se han preocupado por proteger la biodiversidad de la ciudad.

23. Los eventos deportivos internacionales se han preocupado por proteger el patrimonio arquitectónico de la ciudad.

\section{Legado deportivo}

24. Gracias a los eventos deportivos internacionales se ha aumentado la práctica deportiva de los ciudadanos.

25. Gracias a los eventos deportivos internacionales ha aumentado el número de instalaciones deportivas en la ciudad.

26. Gracias a los eventos deportivos internacionales se ha incrementado el apoyo a las organizaciones deportivas de la ciudad.

27. Los eventos deportivos internacionales han dado nuevas oportunidades de practicar deporte a los jóvenes.

28. Los ciudadanos han tenido la oportunidad de participar en la organización de los eventos deportivos internacionales. 


\section{BIBLIOGRAFÍA}

Aces Europa. (2019). American Capitals of Sport. http://aceseurope.eu/ american-capitals-sport/

Aguado, L. F., Osorio, A. M., Arbona, A., \& Peña-Vinces, J. C. (2017). Efectos de la realización de un megaevento deportivo sobre una economía local. El caso de los Juegos Mundiales 2013 Cali. Journal of Economics, Finance and Administrative Science, 22(43), 131-153. https:// doi.org/10.1108/JEFAS-01-2017-0012

Albers, S. (2010). PLS and success factor studies in marketing. En V. Vinzi, W. Chin, J. Henseler, \& H. Wang (Eds.), Handbook of Partial Least Squares (pp. 409-425). Springer.

Aldás, J. (2016). Structural modeling with PLS-SEM: Second order constructs. Esic.

Añó, V., Calabuig, F., \& Parra, D. (2012). Impacto social de un gran evento deportivo: el Gran Premio de Europa de Fórmula 1. (Social impact of a major athletic event: The Formula 1 Grand Prix of Europe). Cultura, Ciencia y Deporte, 7(19), 53-65. https://doi.org/10.12800/ccd.v7i19.23

Azzali, S. (2019). Mega sporting events and their impact on the built environment: Lessons learned from the past. A/Z ITU Journal of the Faculty of Architecture, 16(2), 25-37. https://doi.org/10.5505/itujfa.2019.12499

Boykoff, J. (2017). The Olympics in the Twenty-First Century: Where does Rio 2016 fit in? En A. Zimbalist (Ed.), Rio 2016: Olympic Myths, Hard Realities (pp. 13-34). Brookings Institution Press.

Boykoff, J., \& Zirin, D. (2016). Power Games: A Political History of the Olympics. Verso.

Byers, T., Hayday, E., \& Pappous, A. (Sakis). (2020). A new conceptualization of mega sports event legacy delivery: Wicked problems and critical realist solution. In Sport Management Review (Vol. 23, Issue 2, pp. 171-182). https://doi.org/10.1016/j.smr.2019.04.001

Cashman, R. (2003). What is Olympic Legacy. In M. de Moragas, C. Kennett, \& N. Puig (Eds.), The Legacy of the Olympic Games, 1984-2002 (pp. 31-42). International Olympic Committee.

Cashman, R. (2011). Sydney Olympic park 2000-2010. Walla Walla Press.

Chalip, L. (2006). Towards social leverage of sport events. Journal of Sport and Tourism, 11(2), 109-127. https://doi.org/10.1080/ 14775080601155126

Chalip, L., Green, B. C., Taks, M., \& Misener, L. (2017). Creating sport participation from sport events: making it happen. International Journal of Sport Policy, 9(2), 257-276. https://doi.org/10.1080/19406940 .2016 .1257496

Chappelet, J. (2008). Olympic Environmental Concerns as a Legacy of the Winter Games. The International Journal of the History of Sport, 25(14), 1884-1902. https://doi.org/10.1080/09523360802438991

Chappelet, J. (2012). Mega Sporting Event Legacies: A Multifaceted Concept. Papeles de Europa, 25, 76-86. https://doi.org/10.5209/rev_ pade.2012.n25.41096

Chin, W. (1998). Issues and Opinion on Structural Equation Modeling. MIS Quarterly, 22(1), 6-16.

Chin, W., Marcolin, B., \& Newsted, P. (2003). A Partial Least Squares Latent Variable Modeling Approach for Measuring Interaction Effects: Results from a Monte Carlo Simulation Study and an Electronic-Mail Emotion/Adoption Study. Information Systems Research, 14(2), 189 217. https://doi.org/10.1287/isre.14.2.189.16018

Coalter, F. (2007). A Wider Social Role for Sport: Who's Keeping the Score? Routledge.

Cohen, J. (1988). Statistical Power Analysis for the Behavioral Sciences. Lawrence Erlbaum.

Collins, A., Flynn, A., Munday, M., \& Roberts, A. (2007). Assessing the environmental consequences of major sporting events: The 2003/04 FA Cup Final. Urban Studies, 44(3), 457-476. https://doi. org/10.1080/00420980601131878

Espinal-Ruiz, D., Medina-Vásquez, J., \& Da Cunha-Bastos, F. (2019) Theories, tensions and convergence of sports development. Cultura, Ciencia y Deporte, 14(42), 203-212. https://doi.org/10.12800/ccd. v14i42.1333

Ferranti, E., Andres, L., Denoon-Stevens, S. P., Melgaço, L., Oberling, D. \& Quinn, A. (2020). Operational challenges and mega sporting event legacy: The case of BRT systems in the global south. Sustainability (Switzerland), 12(4). https://doi.org/10.3390/su12041609

Ferreira, L., Lourenção, M., Giraldi, J., \& Oliveira, J. (2018). Economic and image impacts of summer Olympic games in tourist destinations: a literature review. Tourism \& Management Studies, 14(3), 52-63. https://doi.org/10.18089/tms.2018.14305

Flyvbjerg, B. B., Budzier, A., \& Lunn, D. (2020). Regression to the Tail : Why the Olympics Blow Up. Environment and Planning A: Economy and Space, O(0), 1-39. https://doi.org/10.1177/0308518X20958724

Fornell, C., \& Larcker, D. F. (1981). Evaluating Structural Equation Models with Unobservable Variables and Measurement Error. Journal of Marketing Research, 18(1), 39-50. https://doi.org/10.1177/ 002224378101800104

Gaffney, C. (2013). Between discourse and reality: The un-sustainability of mega-event planning. Sustainability (Switzerland), 5(9), 39263940. https://doi.org/10.3390/su5093926

Gehl, J. (2010). Cities for People. Island Press.

Girginov, V. (2012a). Governance of the London 2012 Olympic Games legacy. International Review for the Sociology of Sport, 47(5), 543-558. https://doi.org/10.1177/1012690211413966

Girginov, V. (2012b). The political process of constructing a sustainable London Olympics sport legacy: Three years on. Reflective Practice, 13(3), 483-486. https://doi.org/10.1080/14623943.2012.670110

Girginov, V., \& Hills, L. (2008). A Sustainable Sports Legacy: Creating a Link between the London Olympics and Sports Participation. The International Journal of the History of Sport, 25(14), 2091-2116. https:// doi.org/10.1080/09523360802439015

Girginov, V., \& Hills, L. (2009). The political process of constructing a sustainable London Olympics sports development legacy. International Journal of Sport Policy and Politics, 1(2), 161-181. https://doi. org/10.1080/19406940902950713

Gratton, C., \& Preuss, H. (2008). Maximizing Olympic Impacts by Building Up Legacies. The International Journal of the History of Sport, 25(14), 1922-1938. https://doi.org/10.1080/09523360802439023

Grix, J., Brannagan, P. M., Wood, H., \& Wynne, C. (2017). State strategies for leveraging sports mega-events: unpacking the concept of 'legacy.' International Journal of Sport Policy and Politics, 9(2), 203-218. https://doi.org/10.1080/19406940.2017.1316761

Hair, J., Ringle, C. M., \& Sarstedt, M. (2014). Corrigendum to "Editorial Partial Least Squares Structural Equation Modeling: Rigorous Applications, Better Results and Higher Acceptance" [LRP 46/1-2 (2013) 1-12]. Long Range Planning, 47(6), 392. https://doi.org/10.1016/j. lrp.2013.08.016

Hair, J., Ringle, C., \& Sarstedt, M. (2011). PLS-sem : Indeed a silver bullet. Journal of Marketing Theory and Practice, February 2014. https:// doi.org/10.2753/MTP1069-6679190202

Hallmann, K., Breuer, C., \& Kühnreich, B. (2013). Happiness, pride and elite sporting success: What population segments gain most from national athletic achievements? Sport Management Review, 16(2), 226235. https://doi.org/10.1016/j.smr.2012.07.001

Henseler, J., \& Dijkstra, T. (2020). ADANCO 2.0.0. Kleve: Composite modeling. www.compositemodeling.com

Henseler, J., Hubona, G., \& Ray, P. (2016). Using PLS path modeling in new technology research: Updated guidelines. Industrial Management and Data Systems, 116(1), 2-20. https://doi.org/10.1108/IMDS-092015-0382

Henseler, J., Ringle, C., \& Sarstedt, M. (2015). A new criterion for assessing discriminant validity in variance-based structural equation modeling. Journal of the Academy of Marketing Science, 43(1), 115-135. https://doi.org/10.1007/s11747-014-0403-8

Hiller, H. (2003a). Mega-events, urban boosterism and growth strategies: An analysis of the objectives and legitimations of the Cape Town 2004 Olympic bid. International Journal of Urban and Regional Research, 24(2), 439-458. https://doi.org/10.1111/14682427.00256

Hiller, H. (2003b). Towards a Science of Olympic Outcomes: The Urban Legacy. In M. de Moragas, C. Kennett, \& N. Puig (Eds.), The Legacy of 
the Olympic Games, 1984-2002 (pp. 102-109). International Olympic Committee.

Holmes, K., Hughes, M., Mair, J., \& Carlsen, J. (2015). Events and Sustainability. Routledge.

Höök, K., \& Löwgren, J. (2012). Strong Concepts: Intermediate-Level Knowledge in Interaction Design Research. ACM Transactions on Computer-Human Interaction, 19(3). https://doi.org/10.1145/2362364. 2362371

Hu, L., \& Bentler, P. M. (1998). Fit indices in covariance structure modeling: Sensitivity to underparameterized model misspecification. Psychological Methods, 3(4), 424-453. https://doi.org/10.1037/1082989X.3.4.424

Hyun, M., \& Jordan, J. S. (2020). Athletic goal achievement: A critical antecedent of event satisfaction, re-participation intention, and future exercise intention in participant sport events. Sport Management Review, 23(2), 256-270. https://doi.org/10.1016/j.smr.2019.01.007

International Olympic Committee. (2017). Legacy Strategic Approach Moving Forward. https://www.olympic.org/ /media/Document Library/OlympicOrg/Documents/Olympic-Legacy/IOC_Legacy_Strategy_Full_version.pdf?la=en\%0Ahttps://www.olympic.org/-/media/ Document Library/OlympicOrg/Documents/Olympic-Legacy/IOC_ Legacy_Strategy_Full_version.pdf?la=

Karadakis, K., \& Kaplanidou, K. (2012). Legacy perceptions among host and non-host Olympic Games residents: a longitudinal study of the 2010 Vancouver Olympic Games. European Sport Management Quarterly, 12(3), 243-264. https://doi.org/10.1080/16184742.201 2.680067

Kassens-Noor, E. (2016). From ephemeral planning to permanent urbanism: An urban planning theory of mega-events. Urban Planning, 1(1), 41-54. https://doi.org/10.17645/up.v1i1.532

Kassens-Noor, E., Wilson, M., Müller, S., Maharaj, B., \& Huntoon, L. (2015). Towards a mega-event legacy framework. Leisure Studies, 34(6), 665-671. https://doi.org/10.1080/02614367.2015.1035316

Kim, H., Choe, Y., Kim, D., \& Kim, J. (2019). For sustainable benefits and legacies of mega-events: A case study of the 2018 PyeongChang Winter Olympics from the perspective of the volunteer co-creators. Sustainability (Switzerland), 11(9). https://doi.org/10.3390/su11092473

Kristiansen, E. (2017). Norway. In Elsa Kristiansen, M. Parent, \& B. Houlihan (Eds.), Elite youth sport policy: A comparative analysis (pp. 8095). Routledge.

Latuf de Oliveira Sanchez, R., \& Essex, S. (2018). The challenge of urban design in securing post-event legacies of Olympic Parks. Journal of Urban Design, 23(2), 278-297. https://doi.org/10.1080/13574809. 2017.1395690

Leal de Oliveira, F., Vainer, C. B., Mascarenhas, G., Bienenstein, G., \& Braathen, E. (2020). Mega-events, legacies and impacts: notes on 2016 Rio de Janeiro Olympics. International Journal of Urban Sustainable Development, 12(1), 89-102. https://doi.org/10.1080/1946313 8.2019.1650748

Leopkey, B., \& Parent, M. (2012). Olympic games legacy: From general benefits to sustainable long-term legacy. International Journal of the History of Sport, 29(6), 924-943. https://doi.org/10.1080/09523367 .2011 .623006

Leopkey, B., \& Parent, M. (2015). Stakeholder perspectives regarding the governance of legacy at the Olympic Games. Annals of Leisure Research, 18(4), 528-548. https://doi.org/10.1080/11745398.2015.109 2388

Liu, D., Broom, D., \& Wilson, R. (2014). Legacy of the Beijing Olympic Games: a non-host city perspective. European Sport Management Quarterly, 14(5), 485-502. https://doi.org/10.1080/16184742.2014 .947301

Liu, Y.-D. (2018). Legacy planning and event sustainability: Helsinki as the 2012 World Design Capital. Sustainability (Switzerland), 10(7). https://doi.org/10.3390/su10072453

Ma, S. C., Egan, D., Rotherham, I., \& Ma, S. M. (2011). A framework for monitoring during the planning stage for a sports mega-event. Journal of Sustainable Tourism, 19(1), 79-96. https://doi.org/10.1080/096 69582.2010.502576

Maussier, B. (2017). The New Ethical Dimension of Sports Events: a Reflection on the Evolution from the Ancient Greek Olympic Sports Fes- tival to Postmodern Sports Events. Cultura, Ciencia y Deporte, 12(34), 15-25. https://doi.org/10.12800/ccd.v12i34.828

Méndez, C. P. (2016). Sporting mega-events in the brics countries: Questioning their performance. Revista CIDOB d'Afers Internacionals, 112, 199-223. https://doi.org/10.24241/rcai.2016.112.1.199

Mitrofanova, I. V., Russkova, E. G., Batmanova, V. V., \& Shkarupa, E. A. (2015). Drivers of the regional economic growth and the problem of "White Elephants" of the Russian Olympic megaproject "Sochi 2014." Mediterranean Journal of Social Sciences, 6(4S2), 267-276. https://doi. org/10.5901/mjss.2015.v6n4s2p267

Müller, M. (2015). The mega-event syndrome: Why so much goes wrong in mega-event planning and what to do about it. Journal of the American Planning Association, 81(1), 6-17. https://doi.org/10.1080/01944 363.2015.1038292

Orr, M., \& Inoue, Y. (2019). Sport versus climate: Introducing the climate vulnerability of sport organizations framework. Sport Management Review, 22(4), 452-463. https://doi.org/10.1016/j.smr.2018.09.007

Pappous, A. (2011). Do the Olympic Games lead to a Sustainable Increase in Grassroots Sport Participation? In J. Savery \& G. Gilbert (Eds.), Sustainability and Sport (pp. 81-89). Common Ground. http://kar. kent.ac.uk/31622/

Parent, M. (2016). Stakeholder perceptions on the democratic governance of major sports events. Sport Management Review, 19(4), 402-416. https://doi.org/10.1016/j.smr.2015.11.003

Parent, M., \& Smith-Swan, S. (2012). Managing Major Sports Events: Theory and Practice. Routledge.

Parra, D., Año, V., Calabuig, F., \& Ayora, D. (2016). Residents perceptions about the legacy of America's Cup | Percepción de los residentes sobre el legado de la America's Cup. Cuadernos de Psicologia Del Deporte, 16(1), 325-338.

Preuss, H. (2007). The conceptualisation and measurement of mega sport event legacies. Journal of Sport and Tourism, 12(3-4), 207-228. https://doi.org/10.1080/14775080701736957

Preuss, H. (2011). A method for calculating the crowdingout effect in sport mega-event impact studies: The 2010 FIFA world cup. Development Southern Africa, 28(3), 367-385. https://doi.org/10.1080/037 6835X.2011.595995

Preuss, H. (2015). A framework for identifying the legacies of a mega sport event. Leisure Studies, 34(6), 643-664. https://doi.org/10.1080 /02614367.2014.994552

Preuss, H. (2018). Event legacy framework and measurement. International Journal of Sport Policy and Politics, 11(1), 103-118. https://doi. org/10.1080/19406940.2018.1490336

Preuss, H., Königstorfer, J., \& Dannewald, T. (2020). Contingent Valuation Measurement for Staging the Olympic Games: The Failed Bid to Host the 2018 Winter Games in Munich. In Perspektiven des Dienstleistungsmanagements (pp. 461-478). Springer Fachmedien Wiesbaden. https://doi.org/10.1007/978-3-658-28672-9_23

Pye, P. N., Toohey, K., \& Cuskelly, G. (2015). The social benefits in sport city planning: a conceptual framework. Sport in Society, 18(10), 11991221. https://doi.org/10.1080/17430437.2015.1024235

Rojas, C., \& Espinal-Ruiz, D. (2020). Lineamientos de política pública para juegos y eventos en Colombia. In D. Espinal-Ruiz \& D. Carranza (Eds.), Nuevas Tendencias en la Gerencia Deportiva (pp. 66-80). Escuela sin Fronteras.

Sánchez, J. (2019). Los eventos deportivos como instrumento de desarrollo local. Cultura, Ciencia y Deporte, 14(41), 91-92. https://doi.org/ http://dx.doi.org/10.12800/ccd.v14i41.1268

Smith, A. (2009). Theorising the relationship between major sport events and social sustainability. Journal of Sport and Tourism, 14(2-3), 109-120. https://doi.org/10.1080/14775080902965033

Smith, A. (2010). The development of "sports-city" zones and their potential value as tourism resources for urban areas. European Planning Studies, 18(3), 385-410. https://doi.org/10.1080/09654310903497702

Taks, M., Green, C., Misener, L., \& Chalip, L. (2017). Sport participation from sport events: why it doesn't happen? Marketing Intelligence \& Planning, 36(2), 185-198. https://doi.org/10.1108/MIP-05-20170091

Teo, T. S. H., Srivastava, S. C., \& Jiang, L. (2008). Trust and Electronic Government Success: An Empirical Study. Journal of Management In- 
formation Systems, 25(3), 99-132. https://doi.org/10.2753/MIS0742 1222250303

Thomson, A., Cuskelly, G., Toohey, K., Kennelly, M., Burton, P., \& Fredline, L. (2019). Sport event legacy: A systematic quantitative review of literature. Sport Management Review, 22(3), 295-321. https://doi. org/10.1016/j.smr.2018.06.011

Toledo, R. M., Grix, J., \& Bega, M. T. S. (2015). Megaeventos esportivos e seus legados: Uma análise dos efeitos institucionais da eleição do Brasil como país-sede. Revista de Sociologia e Politica, 23(56), 21-44. https://doi.org/10.1590/1678-987315235602

Tsao, W. C., Hsieh, M. T., \& Lin, T. M. Y. (2016). Intensifying online loyalty! the power of website quality and the perceived value of consumer/ seller relationship. Industrial Management and Data Systems, 116(9), 1987-2010. https://doi.org/10.1108/IMDS-07-2015-0293

Valaei, N., \& Jiroudi, S. (2016). Job satisfaction and job performance in the media industry: A synergistic application of partial least squares path modelling. Asia Pacific Journal of Marketing and Logistics, 28(5), 984-1014. https://doi.org/10.1108/APJML-10-2015-0160

Wicker, P. (2019). The carbon footprint of active sport participants. Sport Management Review, 22(4), 513-526. https://doi.org/10.1016/j. smr.2018.07.001

Wilson, W. (2015). Sports infrastructure, legacy and the paradox of the 1984 olympic games. International Journal of the History of Sport, 32(1), 144-156. https://doi.org/10.1080/09523367.2014.986110 